Artículo científico

Volumen 30(2):425-436. Mayo-agosto, 2019

e-ISSN 2215-3608, doi:10.15517/am.v30i2.33574

https://revistas.ucr.ac.cr/index.php/agromeso/index

\title{
Correlaciones alométricas en Hylocereus costaricensis y H. monocanthus (pitahaya): una herramienta para cuantificar el crecimiento ${ }^{1}$
}

\section{Allometric correlation in Hylocereus costaricensis y H. monocanthus (pitahaya): a tool to quantify growth}

\author{
Gabriel Garbanzo-León ${ }^{2}$, Greddin Chavarría-Pérez ${ }^{3}$,Edgar V. Vega-Villalobos ${ }^{4}$
}

1 Recepción:13 de junio, 2018. Aprobación: 13 de noviembre, 2018. Este trabajo formó parte de los proyectos de investigación VI 520 B6 022 y VI 520 B7 004 inscritos en Vicerrectoría de Investigación de la Universidad de Costa Rica, San José, Costa Rica.

2 Universidad de Costa Rica, Escuela de Agronomía, Centro de Investigaciones Agronómicas, Sabanilla, San José. C.P. 11501, San José, Costa Rica. juan.garbanzo@ucr.ac.cr (autor para correspondencia; https://orcid.org/0000-0003-2848-6199)

3 Universidad de Costa Rica, Escuela de Agronomía, Finca Experimental de Santa Cruz, Sede de Guanacaste, Costa Rica. greddi2@live.com

4 Universidad de Costa Rica, Escuela de Agronomía, Departamento de Cultivos, San José, Costa Rica. edgar.vega@ucr.ac.cr

\section{Resumen}

Introducción. La pitahaya es una planta que pertenece a la familia de las cactáceas, esta crece naturalmente en árboles o tutores muertos en zonas estacionalmente secas y posee una arquitectura variable en su crecimiento por ser una planta epífita y hemiepífita, sus tallos verdes son estructuras que cumplen un papel de tallo-hoja. Actualmente en Costa Rica existe poca información sobre el crecimiento y la cuantificación de la morfometría de la pitahaya. Objetivo. El objetivo de este trabajo fue relacionar variables no destructivas con variables destructivas para construir una herramienta para el uso de proyecciones de crecimiento en pitahaya en Costa Rica. Materiales y métodos. El trabajo se efectuó entre los años 2016-2017. Se muestrearon al azar tallos provenientes de plantas sembradas en suelo y sustratos. Se evaluó: largo, grosor basal, medio y alto (A), área fotosintética (método indirecto) y biomasa (peso fresco, seco y porcentaje de humedad) para cada tallo. Se construyó una base de datos y se calcularon regresiones lineales, asimismo, se comparó con la prueba de Kruskal Wallis $(\alpha=0,05)$ la variabilidad entre sitios (suelo arcilloso, franco y viveros) de siembra. Resultados. Las regresiones lineales mostraron que el largo por el ancho presentó una correlación significativa $\left(r^{2}=0,85\right)$ con la variable peso seco y una correlación altamente significativa $\left(r^{2}=0,97\right)$ con la variable área fotosintética. Se determinaron dos criterios matemáticos para generar ecuaciones de mejor ajuste con el fin de lograr una mayor precisión en las proyecciones de las variables. El peso mostró diferencias significativas $(\alpha=0,01)$ según los sitios de crecimiento del tallo. Conclusión. Las regresiones lineales mostraron alta precisión $\left(r^{2}>0,85\right)$ en algunas variables de crecimiento en los tallos de pitahaya, lo cual podría ser una herramienta eficaz para generar cuantificaciones de crecimiento en el cultivo a partir de un parámetro alométrico.

Palabras clave: tallos, cactaceae, ecuaciones, análisis de regresión, Hylocereus.

\begin{abstract}
Introduction. Dragon fruit plant (Pitahaya) is a cactacea family plant, it grows naturally in dead trees or stakes in seasonally dry areas, and it has an out-of-order architecture in the growth for being and epiphyte and hemiepiphyte plant, its green steams are structures that play the role of steam-leaf. Currently, in Costa Rica, there is little information
\end{abstract}


on the growth and quantification of dragon fruit morphometry. Objective. The objective of this work was to relate non-destructive variables with destructive variables to develop a useful tool for the use growth projections in dragon fruit in Costa Rica. Materials and methods. The study was carried from 2016 to 2017. Stems from plants sown in soil and substrates were randomly sampled. The following were evaluated: longitude, basal, medium and apical thickness (A), leaf area (indirect method) and biomass (fresh weight, dry weight and moisture percentaje) for each stem. A database was built and it's linear regressions were calculated, and the site variability (clay soil, loam soil and nurseries) was compared with Kruskal Wallis $(\alpha=0.05)$ test. Results. Linear regressions showed highly significant correlation $\left(r^{2}=0.97\right)$ with the significative correlation $\left(r^{2}=0.85\right)$ with dry weight and that the longitude by width presented a foliar area. Two mathematical criteria were determined, to generate better fit equation for more accurate projections of variables. The weight showed significative differences $(\alpha=0.01)$ according to stem growth sites. Conclusion. Linear regression showed high precision $\left(r^{2}=0.85\right)$ in some growth variables in dragon fruit steams, wich could be an effective tool to generate crop growth quantification.

Keywords: stem, cactaceae, equations, regression analysis, Hylocereus.

\section{Introducción}

La pitahaya es un cultivo perenne que pertenece al orden Caryophyllales y la familia de Cactaceae. Fue descubierta por primera vez en forma silvestre en México, Colombia y Centro América por los españoles, quienes le dieron el nombre de "pitahaya" que significa fruta con escamas (Flores, 2011). Las cactáceaes están compuestas por unos 130 géneros, de los cuales se han descrito entre 1500 y 1800 especies en América (Hunt et al., 2006; Barthlott et al., 2010; Esquivel y Araya, 2012). Los géneros más cultivados a nivel mundial son Hylocereus y Selenicereus, los cuales son nativos de regiones tropicales de América del Norte y Sur.

Las producciones agrícolas de la pitahaya se establecen principalmente en zonas estacionalmente secas, debido a que es una planta que resiste el déficit hídrico (Yuqing et al., 2015). Los países: Vietnam, Israel, India, Nicaragua, Colombia, México y Guatemala, han establecido la especie $H$. undatus que es de mayor rendimiento agronómico (10-45 t.ha' año $^{-1}$ ), pero varía según las condiciones y manejos de cada zona productora (Mizrahi

et al., 1997; Raveh et al., 1998; Sabino, 2010; Tuan, 2012; Mizrahi, 2014; Mercado-Silva, 2018). La pitahaya es conocida en Vietnam como fruta dragón o bien "dragon fruit" en su nombre en inglés, para exportación. Los mayores compradores de su fruta son: China, Corea del Sur, Indonesia, Malasia, Singapur, Holanda, Reino Unido, Francia, Alemania, Estados Unidos y Canadá; siendo China el mayor comprador de la pitahaya roja de Vietnam (Tuan, 2012). Además, la pitahaya roja también se comercializa en Centroamérica y México (Juárez et al., 2001), y la amarilla en Colombia, Bolivia, Ecuador, Perú, Venezuela y Centroamérica (López y Guido, 2002; Flores, 2011). En Costa Rica la actividad agrícola de la pitahaya registrada se muestra en pequeña escala, se cultiva en regiones con limitaciones del recurso hídrico, principalmente en Puntarenas y Guanacaste (García y Quirós, 2010).

Las especies $H$. undatus (roja) y $S$. megalanthus (amarilla) son las que se siembran en mayor escala a nivel mundial (Le-Bellec y Vaillant, 2011; Esquivel y Araya, 2012; Mizrahi, 2014; Morillo et al., 2017). En Costa Rica se identificaron tres especies silvestres: H. costaricensis, H. stenoptrus y H. monocanthus (Bauer, 2003). Otros autores han identificado en Centroamérica genotipos con el nombre de: Orejona, Rosa, Cebra, Lisa, San Ignacio, Nacional y Crespa, las cuales son muy distintas en sus características fenotípicas (Vaillant et al., 2005). Estudios realizados en caracterización morfológica de genotipos de pitahaya, determinaron que estas pertenecen a las especies $H$. costaricensis y H.monocanthus, siendo estas las que se encuentran en las zonas productoras de pitahaya en Costa Rica (López y Guido, 1998; Esquivel et al., 2007).

La planta posee una arquitectura de crecimiento variable, por ser una especie que crecen de forma epífita o hemiepífita (Andrade et al., 2006; Ortiz-Hernández y Carrillo-Salazar, 2012; Mizrahi, 2014). Se desarrolla 
naturalmente en los árboles y tutores muertos, esto hace que no tenga un comportamiento definido de crecimiento debido a que se ajusta al lugar donde se establece. Los tallos verdes (cladodios) son una estructura que cumple la función de tallo-hoja, debido a que tienen la capacidad de fotosintetizar y de sostén de la planta (Gunasena et al., 2007; Huachi et al., 2015; Montesinos et al., 2015; Correia et al., 2017). Los géneros Hylocereus y Selenicereus (cultivadas) presentan tallos con tres aristas (costillas) o caras (en ocasiones 4), lo cual tiene una configuración en forma de triángulo longitudinalmente (Mizrahi, 2014; Montesinos et al., 2015). La planta presenta varios órdenes de tallos, primarios, secundarios, terciarios y cuaternarios, definidos por su tiempo de crecimiento y brotación (Suárez, 2011); además, presentan una estructura leñosa en el centro, con una dureza relativamente alta que incrementa con la edad y el posicionamiento en la planta (tallo basal, tallos primarios, secundarias entre otros). Tienen un crecimiento indefinido, debido a que se pueden encontrar tallos muy largos o cortos según su brotación (Balaguera-López et al., 2010); en ocasiones el crecimiento se define por la pérdida de la dominancia apical, lo que implica un estímulo específico en el tallo, que provoca nuevas brotaciones de yemas (Cerqueda, 2010; Viñas et al., 2012).

En la actualidad existe poca información con relación al crecimiento y la morfometría de los tallos en $H$. costaricensis y H. monocanthus. Algunos estudios realizados en veintiún genotipos de pitahaya se encontraron diferencias entre ellos, principalmente por las características del tallo para la especie H. undatus (Grimaldo-Juárez et al., 2007). En genotipos de S. megalanthus (pitahaya amarilla) se encontraron diferencias morfológicas en la cantidad de espinas, aréolas, altura de la aréola, cera, pigmentación en los filocladodios, textura, entre otros, donde estas características están en función de los sitios donde se establecieron las plantaciones (Morillo et al., 2017). Las características son variables, las cuales se definen por el manejo del cultivo, debido a las diversas técnicas que existen (Juárez et al., 2009; Mejía et al., 2013; Cálix et al., 2014). En general, la información existente se enfoca en la caracterización del fruto (Juárez et al., 2001; Castillo et al., 2005; Esquivel et al., 2007; Esquivel y Araya, 2012) y no en los tallos o características fenotípicas de la planta. A esto se suma la escasa información y la creación de herramientas para la cuantificación del crecimiento del cultivo mediante variables no destructivas a partir de variables morfológicas de los tallos. En los cultivos perenes el desarrollo de herramientas alométricas es eficaz para las proyecciones de producción y crecimiento (Fonseca et al., 2013; Rojas y Seminario, 2014), con el fin de generar información ajustada para el manejo y las proyecciones del cultivo. El objetivo de este trabajo fue relacionar variables no destructivas con variables destructivas para construir una herramienta para el uso de proyecciones de crecimiento en pitahaya en Costa Rica.

\section{Materiales y métodos}

Este trabajo se realizó entre enero de 2016 y octubre de 2017 en la Finca Experimental de Santa Cruz (FESC) de la Universidad de Costa Rica, Santa Cruz, Costa Rica. Se obtuvieron tallos del cultivo y viveros sembrados en

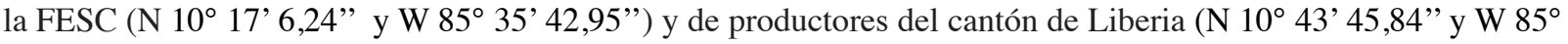
$\left.25^{\prime} 31,86^{\prime \prime}\right)$, ambos lugares ubicados en Guanacaste. La precipitación promedio anual fue de $1834 \mathrm{~mm} \cdot \mathrm{año}^{-1}$ y la temperatura media anual de $27,9^{\circ} \mathrm{C}$.

Las características de los suelos y los sustratos donde se recolectaron los tallos fueron variables, según las características fisicoquímicas y épocas climáticas del año. Es por esta razón que este experimento clasificó cuatro sitios de siembra distintos (Cuadro 1). El primer sitio (Sustrato 1) fueron plantas sembradas en un vivero en época seca (enero-mayo), en un sustrato con una mezcla volumétrica de $40 \%$ granza más $60 \%$ suelo. El segundo sitio (Sustrato 2) también fueron plantas sembradas en viveros, pero en época lluviosa (agosto-noviembre) con la misma mezcla volumétrica. Para ambos viveros se sembraron en bolsas de 1,5 1, bajo una casa de mallas abierta, con riego por aspersión y con un sarán de 30 \% de sombra en la FESC, según lo recomendado por Reveh et al. (1998). El 
Cuadro 1. Características de los sitios de crecimiento del cultivo de pitahaya (H. costaricensis y H. monocanthus) en Guanacaste, Costa Rica. 2017.

Table 1. Sites characteristics of dragon fruit (H. costaricensis y H. monocanthus) crop growth in Guanacaste, Costa Rica. 2017.

\begin{tabular}{cccc}
\hline Sitio & Sistema de siembra & Tipo o textura & Lugar \\
\hline Sustrato 1 & Vivero en época seca* & $40 \%$ granza $+60 \%$ suelo & Infraestructura con sarán \\
Sustrato 2 & Vivero en época lluviosa** & $40 \%$ granza $+60 \%$ suelo & Infraestructura con sarán \\
Suelo 1 & Suelo & Franco arcillo arenosa & Plantación Liberia \\
Suelo 2 & Suelo & Arcillosa & Plantación Santa Cruz \\
\hline
\end{tabular}

* Época seca: temporada de verano entre enero y mayo de cada año en la zona de Guanacaste. ** Época lluviosa: temporada de agosto y noviembre de cada año en la zona de Guanacaste / *Dry season: summer season between January and May of each year in the Guanacaste area.** Rainy season: winter season between August and November of each year in the Guanacaste area.

tercer sitio (Suelo 1) eran tallos provenientes de una plantación sembrada en Liberia, Guanacaste en un Entisol franco arcillo arenoso, suelo con alta friabilidad, con una capacidad de intercambio catiónico efectiva (CICE) de $11,7 \mathrm{cmol}(+) .1^{-1}, \mathrm{pH}$ de 5,2 , altos contenidos de fósforo (P) $\left(78 \mathrm{mg} . \mathrm{l}^{-1}\right)$ y con presencia de acidez intercambiable $\left(0,95 \mathrm{cmol}(+) \cdot \mathrm{l}^{-1}\right)$. El último sitio (Suelo 2) perteneció a una plantación sembrada en la FESC, en un suelo Alfisol arcilloso con características vérticas, con una CICE de 29,6 cmol(+).1-1, $\mathrm{pH}$ de 5,5, bajos contenidos de P $\left(1 \mathrm{mg} . .^{-1}\right)$ y sin presencia de acidez (0,11 cmol(+).1-1 $)$ (Guzmán, 1987; Salazar, 1991; Vega y Salas, 2012).

Los tallos se seleccionaron al azar de plantas de las especies $H$. costaricensis y H. monocanthus, ambas de frutas de color rojo y de plantaciones abiertas con polinización al azar. Se utilizaron tallos de distintos grosores, tamaños y pesos con el fin de garantizar una heterogeneidad. Las muestras fueron procesadas en la FESC, donde se midieron los parámetros alométricos, área fotosintética y pesos.

\section{Determinación de largo $\mathrm{x}$ ancho}

Para evaluar el ancho del tallo, primero se observó cada detalle en la estructura de la planta. Al presentar una configuración en forma de triángulo, los tallos presentaron un arreglo de tres aristas (en ocasiones cuatro), lo cual divide el tallo en tres aristas gruesas y de distinto grosor en toda su longitud (Montesinos et al., 2015). Se midió el grosor en las tres aristas, la primera medición se efectuó en la parte basal de la arista 1, en la segunda, la parte media de la arista 2 y la tercera en la parte alta (meristemo apical) de la arista 3 (Figura 1). Se midió el largo desde la parte basal hasta el meristemo apical del tallo. Por último, para determinar el largo por el ancho se aplicó la ecuación 1.

\section{$\frac{\sum \text { ancho de caras }}{\text { número de caras }} \times$ longitud del tallo (cladodio $)=L x A$}

ecuación 1

\section{Área fotonsitética de los tallos}

La medición del área foliar de los tallos se realizó por medio de una medida indirecta, usando fotografías digitales con una cámara modelo Olympus SZ-30MR (16 megapixeles). Para cada tallo se tomaron tres fotografías, específicamente en cada cara, y se midió la longitud de este para brindar una escala (en cm) específica. 


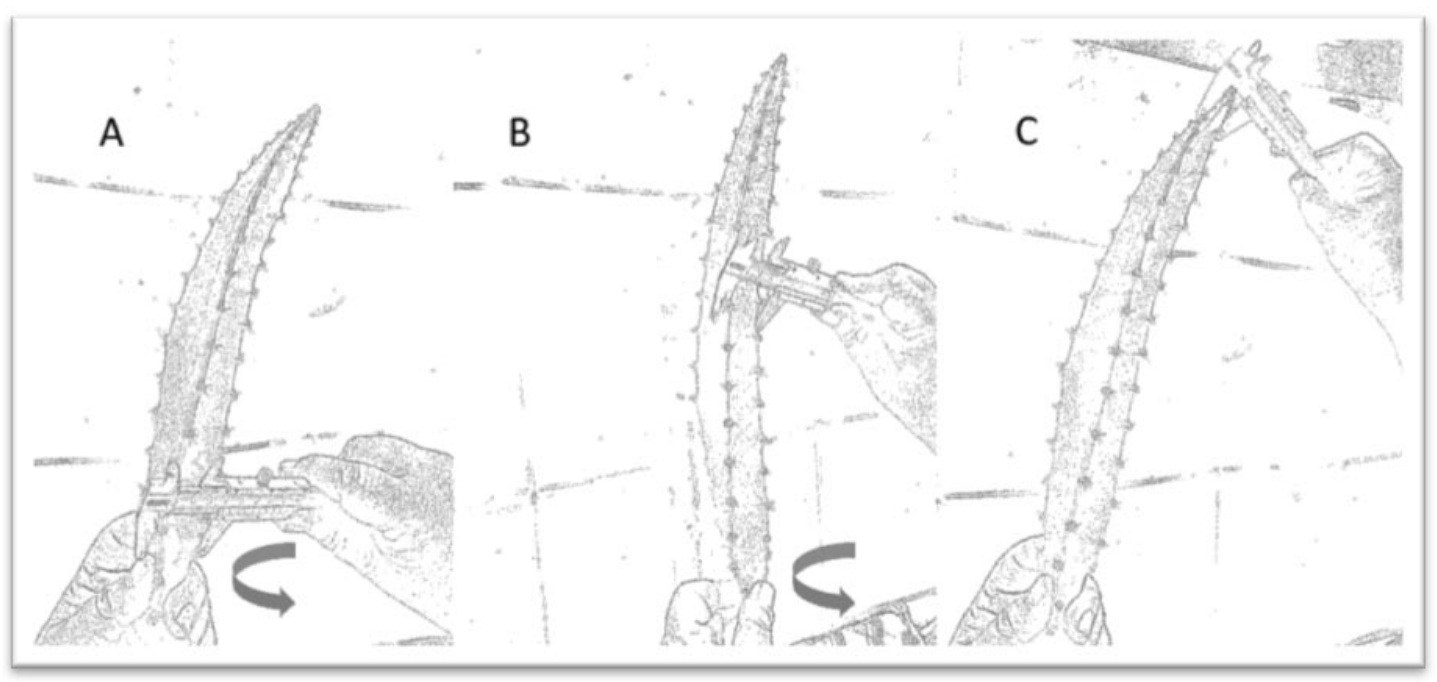

Figura 1. Cuantificación ascendente del grosor del tallo de pitahaya en tres distintas aristas; A) grosor basal "arista 1"; B) rotación y grosor medio "arista 2"; C) rotación y grosor apical "arista 3", en una zona estacionalmente seca. Costa Rica. 2017.

Figure 1. Ascending quantification of the thickness of the dragon fruit stem in three different edges; A) basal thickness "edge 1"; B) rotation and medium thickness "edge 2"; C) rotation and apical thickness "edge 3", in the seasonally dry zone. Costa Rica. 2017.

Posteriormente, las fotografías se analizaron mediante el programa ImageJ versión 1.50i, según la metodología de Rincón et al. (2012) y validada por Newton et al. (2013). Se determinó el área para cada cara del tallo y se calculó la sumatoria de las tres caras para estimar el área fotosintéticamente activa total en cada estructura.

\section{Determinación de pesos}

Los tallos fueron identificados para las correlaciones respectivas, a cada uno se le midió el peso fresco, se fraccionaron en estructuras más pequeñas y se secaron durante $48 \mathrm{~h} \mathrm{a} 80^{\circ} \mathrm{C}$. Luego se pesaron nuevamente para determinar el peso seco de cada uno. Una vez obtenidos ambos pesos de las muestras, se calculó el porcentaje de humedad presente en cada estructura.

\section{Análisis de los datos}

Se realizaron bases de datos para correlacionar el LxA (largo por ancho) con el área fotosintética, peso seco y peso fresco (P.F) evaluados para cada tallo. Asimismo, se desarrolló una base de datos específica para el efecto de distintos sitios de siembra en comparación a la humedad de cada tallo. Una vez completas las bases se procedió a calcular regresiones lineales para la correlación del LxA para un número (n) de 216 observaciones; también, se realizó un análisis no paramétrico mediante la prueba estadística Kruskal Wallis $(\alpha=0,05)$. Para ajustar las regresiones a una correlación más precisa y validada, se desarrollaron criterios específicos para las variables en el eje "X” (LxA y P.F). Todos los datos fueron calculados mediante el software estadístico Rstudio versión 1.0.136. 


\section{Resultados}

Las regresiones lineales mostraron un buen ajuste $(>0,85)$ para algunas variables morfológicas (Figura 2). El largo por el ancho de tallo mostró una correlación significativa $\left(\mathrm{r}^{2}=0,85\right)$ con la variable de peso seco, y una correlación altamente significativa $\left(\mathrm{r}^{2}=0,97\right)$ con la variable área foliar. Sin embargo, el largo por el ancho del tallo presentó ajustes medios $\left(\mathrm{r}^{2}=0,57\right)$ con relación al peso fresco. Este mismo comportamiento $\left(\mathrm{r}^{2}=0,62\right)$ se encontró cuando se correlacionó el peso fresco con el peso seco.
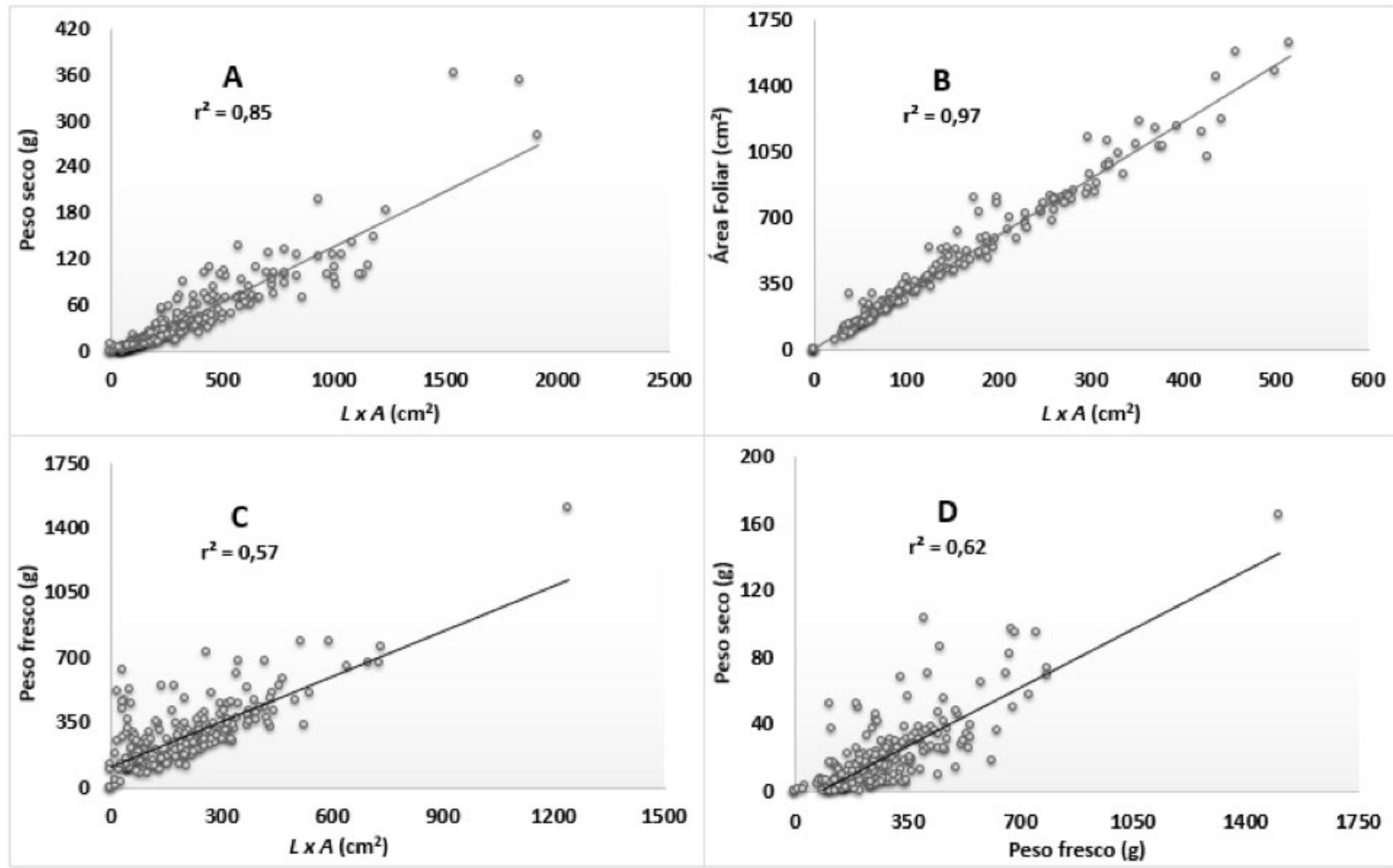

Figura 2. Regresiones lineales modeladas entre el largo por ancho (LxA), peso fresco y distintas variables determinadas en tallos de pitahaya (H. costaricensis y H. monocanthus), medidos en el trópico con estación seca. Costa Rica. 2017.

A) Regresión lineal entre LxA y peso seco, $\mathrm{n}=291$; B) Regresión lineal entre LxA y área foliar del tallo, n =197; C) Regresión lineal entre LxA y el peso fresco en tallos, n=210; D) Regresión lineal entre el peso fresco y el peso seco, n=216.

Figure 2. Linear regressions modeled between lenght by width (LxA), fresh weight and different variables determined in dragon fruit steams (H. costaricensis and H. monocanthus), measured in the tropic with dry season, Costa Rica, 2017.

A) Linear regression between LxA and dry weight, $n=291$; B) linear regression between LxA and leaf area steam, n=197; C) linear regression between LxA and fresh weight in steams, $n=210$; D) linear regression between dry and fresh weight, $n=216$.

Se encontraron dos modelos de mejor ajuste según criterios generados y validados para cada variable (Cuadro 2). Se determinó que el modelo 1 se ajustó mejor a una ecuación donde se estimó un intercepto " 0 ” ( $\mathrm{y}=\mathrm{bx}$ ), mientras que, el modelo 2 mostró un mejor ajuste a una regresión lineal simple (y=a+bx). Se encontró que, para la regresión 
Garbanzo-León et al.: Proyecciones alométricas en pitahaya

Cuadro 2. Criterios para utilizar las ecuaciones de mejor ajuste para generar predicciones según la variable alométrica LxA y peso fresco en tallos de pitahaya (H. costaricensis y H. monocanthus), del trópico con estación seca, Guanacaste, Costa Rica. 2017.

Table 2. Criteria to use the best fitting equations to generate predictions according to the allometric variable LxA and fresh weight in stems of pitahaya (H. costaricensis and H. monocanthus), from the tropics with dry season, Guanacaste, Costa Rica. 2017.

\begin{tabular}{ccccccc}
\hline Figura 2 & \multicolumn{3}{c}{ Modelo 1 } & \multicolumn{3}{c}{ Modelo 2 } \\
\cline { 2 - 7 } & Criterio & Ecuación & $\mathbf{r}^{2}$ & Criterio & Ecuación & $\mathbf{r}^{2}$ \\
\hline $\mathrm{A}$ & $\mathrm{LxA} * \leq 58 \mathrm{~cm}^{2}$ & $\mathrm{Y}=0,1313 \mathrm{x}$ & 0,83 & $\mathrm{LxA} \geq 59 \mathrm{~cm}^{2}$ & $\mathrm{Y}=0,145126 \mathrm{x}-8,19287$ & 0,85 \\
$\mathrm{~B}$ & $\mathrm{LxA} \leq 38 \mathrm{~cm}^{2}$ & $\mathrm{Y}=3,0475 \mathrm{x}$ & 0,97 & $\mathrm{LxA} \geq 39 \mathrm{~cm}^{2}$ & $\mathrm{Y}=3,00074 \mathrm{x}+10,76532$ & 0,97 \\
$\mathrm{C}$ & $\mathrm{LxA} \leq 337 \mathrm{~cm}^{2}$ & $\mathrm{Y}=1,1532 \mathrm{x}$ & 0,42 & $\mathrm{LxA} \geq 338 \mathrm{~cm}^{2}$ & $\mathrm{Y}=0,8175 \mathrm{x}+113,21$ & 0,57 \\
$\mathrm{D}$ & $\mathrm{P} . \mathrm{F}^{* *} \leq 393 \mathrm{~g}$ & $\mathrm{Y}=0,0817 \mathrm{x}$ & 0,59 & $\mathrm{P} . \mathrm{F} \geq 394 \mathrm{~g}$ & $\mathrm{Y}=0,0997 \mathrm{x}-7,0878$ & 0,62 \\
\hline
\end{tabular}

* Multiplicación del largo por el ancho. ** peso fresco / * Process of multiplication of the stem length by stem width. ** wet weight.

LxA vs peso seco, se debe de utilizar el criterio $\mathrm{LxA} \leq 58 \mathrm{~cm}^{2}$ para el modelo 1 , mientras que, para el modelo 2 el criterio $\mathrm{LxA} \geq 59 \mathrm{~cm}^{2}$, con $\mathrm{r}^{2}$ de 0,83 y 0,85 , respectivamente. La regresión LxA vs área fotosintética se ajustó para el modelo 1 el criterio LxA $\leq 38 \mathrm{~cm}^{2}$, mientras que, para el modelo 2 el criterio LxA $\geq 39 \mathrm{~cm}^{2}$, con un $\mathrm{r}^{2} \mathrm{de}$ 0,97 para ambos. En la comparación entre LxA vs peso fresco se encontró que el modelo 1 se ajustó a un criterio LxA $\leq 337 \mathrm{~cm}^{2}$ con $\mathrm{r}^{2}$ de 0,42 , mientras que, para el modelo 2 el criterio LxA $\geq 338 \mathrm{~cm}^{2}$, con un $\mathrm{r}^{2}$ de 0,57 , brindó un mejor resultado. Para la correlación entre peso fresco (P.F) y peso seco, se encontró que en el modelo 1 se debe utilizar el criterio P.F $\leq 393$ g, mientras que, para el modelo 2 el criterio P.F $\geq 394$ g, los cuales presentaron un $\mathrm{r}^{2}$ ajustado entre 0,59 y 0,62 , respectivamente.

Se cuantificó estadísticamente que los tallos usados para las regresiones variaron con relación a peso y humedad (Cuadro 3). Se encontró diferencias estadísticamente significativas $(\alpha<0,01)$ en el peso fresco de los

Cuadro 3. Comparaciones de sitio de siembra sobre peso fresco, peso seco y humedad de los tallos de pitahaya (H. costaricensis y H. monocanthus) en una zona con estación seca, en Guanacaste, Costa Rica. 2017.

Table 3. Planting sites comparisons in the fresh weight, dry weight, and moisture of the dragon fruit steams (H. costaricensis and H. monocanthus), in an area with dry season, Guanacaste, Costa Rica. 2017.

\begin{tabular}{lccc}
\hline Sitio & Peso fresco & Peso seco & Humedad \\
\cline { 2 - 4 } & & Rangos de comparaciones \\
\hline Sustrato $1 *$ & $(221) 82 \mathrm{c}$ & $(5) 43 \mathrm{c}$ & $(98) 178 \mathrm{a}$ \\
Sustrato $2 * *$ & $(254) 95 \mathrm{bc}$ & $(17) 104 \mathrm{~b}$ & $(93) 109 \mathrm{~b}$ \\
Suelo $1 * * *$ & $(345) 138 \mathrm{a}$ & $(29) 151 \mathrm{a}$ & $(92) 65 \mathrm{c}$ \\
Suelo 2**** & $(369) 125 \mathrm{ab}$ & $(48) 166 \mathrm{a}$ & $(86) 31 \mathrm{~d}$ \\
Shapiro test & $<0,001$ & $<0,001$ & $<0,001$ \\
Kruskal Wallis & $<0,001$ & $<0,001$ & $<0,001$ \\
\hline
\end{tabular}

* tallos obtenidos de viveros en época seca sembrados en sustrato (40\% granza $+60 \%$ suelo) / nurseries stems planted in mixtures substrates $(40 \%$ rice husk $+60 \%$ soil) in dry season. $* *$ tallos obtenidos de viveros en época lluviosa sembrados en sustrato $(40 \%$ granza $+60 \%$ suelo) / stems obtained from nurseries in the rainy season planted in substrate (40\% rice husk $+60 \%$ soil). $* * *$ tallos obtenidos de plantaciones sembradas en suelos franco arcillo arenosos / stems obtained from plantations planted in sandy clay loam soils. **** tallos obtenidos de plantaciones sembradas en suelos arcillosos / crops stems planted in clay soil.

Shapiro Wilk: Prueba de normalidad. Kruskal Wallis: prueba no paramétrica. Medias seguidas por una misma letra no representa diferencias significativas según la prueba no paramétrica Kruskal Wallis a una probabilidad de $5 \%$ ( $<<0,05)$. Datos en paréntesis representan observaciones reales para el peso (gramos) y para \% de humedad / Shapiro Wilk: Normality test. Kruskal Wallis: nonparametric test. Means followed by the same letter does not represent significant differences according to the nonparametric Kruskal Wallis test at a $5 \%$ probability $(\mathrm{p}<0.05)$. Data in parentheses represent actual observations for weight (grams) and for $\%$ moisture. 
tallos sembrados directamente en el suelo en comparación a los tallos sembrados en el sustrato. Este mismo comportamiento se presentó para el peso seco, sin embargo, para la humedad contenida en las estructuras, la significancia $(\alpha<0,001)$ fue mayor en las plantas sembradas en sustratos, que en el suelo (Cuadro 3 ).

\section{Discusión}

Las regresiones basadas en el LxA son una herramienta precisa para la cuantificación no destructiva de variables de crecimiento. Los criterios generados en el modelo 1 y 2 brindan un mayor ajuste y precisión a datos según el tamaño del LxA y el peso fresco (P.F) en cada tallo. Es importante realizar el uso del modelo 1 cuando los datos del LxA son bajos y el modelo 2 cuando los valores del LxA son altos. El uso de herramientas alométricas está demostrado en cultivos y forestales para la estimación de biomasa (Acosta-Mireles et al., 2002; López-Merlín et al., 2003; Fonseca et al., 2013) y área foliar (Galindo y Clavijo, 2007; Rojas y Seminario, 2014) en evaluaciones no destructivas (Nygren et al., 1993).

El área fotosintética fue la variable que más se ajustó a las regresiones. La evaluación mediante la metodología de Rincón et al. (2012) se ajustó bien a la estimación de área foliar en pitahaya. Esto fue mostrado por los coeficientes encontrados $\left(r^{2}=0,97\right)$ y al poseer un menor número $(n=197)$ de observaciones en campo para ajustar los modelos. Las variables de peso presentaron una gran variación y un menor ajuste con la variable LxA, es posible que este efecto sea producto del crecimiento de las plantas en distintos sistemas de siembra y el sitio donde fueron plantadas (Cuadro 1). La variabilidad en el crecimiento con relación al contenido relativo de agua en plantas de pitahaya está demostrada por Yuqing et al. (2015), quienes encontraron que a deficiencias de agua $(<40 \%)$, la planta no puede eliminar sustancias tóxicas como el malondialdehído. En Guanacaste, Costa Rica, existen temporadas muy secas y lluviosas, provocando a las plantas un acelerado crecimiento en las épocas lluviosas, mismo que disminuye en épocas secas. Sin embargo, para este trabajo la variabilidad en el crecimiento de los tallos fue esencial para el desarrollo de esta herramienta, porque se podía ajustar a ecuaciones para los cultivos de pitahaya sembrados en las condiciones del trópico seco de Costa Rica.

Las mediciones del largo y ancho en un tallo también mostraron gran variabilidad, debido a que estas estructuras presentan una arquitectura en forma de triángulo, las cuales mostraron, en la mayoría de las ocasiones, tres áreas fotosintéticamente activas (Mizrahi, 2014; Montesinos et al., 2015). Según el posicionamiento del tutor en la planta, el grado de sombra (luminosidad), estado fenológico y la especie cultivada, pueden presentarse distintos grosores o tamaños en las aristas (Raveh et al., 1998; Kishore, 2016), asimismo, otras investigaciones han demostrado que las dimensiones de los tallos pueden verse influenciadas por el manejo de la fertilización en las plantaciones (Mizrahi, 2014; Fumuro, 2016).

El grosor del tallo varía en toda su longitud, por lo cual la metodología de rotación y medición de las caras, posiblemente sea la medida más idónea del grosor. Entre más mediciones del grosor del tallo, se podría obtener un dato más exacto, sin embargo, demandaría gran cantidad de tiempo y mayor cantidad de cálculos para los productores. Caso contrario, si se realiza solo una medición en un segmento y en una arista podría sobreestimar el ancho real que presenta el tallo, por lo cual, estas ecuaciones no se ajustarían bien a las predicciones de área foliar y pesos.

La variación del sistema de siembra y la textura del sitio de siembra de los tallos mostraron una variación importante del crecimiento y la humedad. Se encontró que el peso seco y fresco cambió según la característica del sitio de siembra, sin embargo, esto es de esperar, debido a que se obtuvieron tallos de distintos tamaños y dimensiones. El porcentaje de humedad en estos cambian significativamente $(\alpha<0,01)$ con el sitio de siembra, pero en este caso no debería variar según el tamaño del tallo, porque es una variable que se mide específicamente para cada individuo. Se encontró que los tallos muestreados en suelos de textura arcillosa mostraron menor porcentaje de 
humedad en su estructura que los obtenidos en suelos francos y en sustratos. Es probable que los tallos desarrollados en suelos franco y sustratos requieran mayor almacenamiento de agua, por lo cual, es posible que este efecto sea producto de la retención de agua en el suelo y sustrato, debido a que los suelos francos retienen menos cantidad de agua que los suelos arcillosos, donde estos últimos podría sostener más humedad (no disponible para la planta) por más tiempo (Sellés et al., 2003; Martín y Muñoz, 2017). Estos datos son solo una proyección al comportamiento de almacenamiento de agua en la estructura, lo cual requiere de mayor análisis para determinar esta tendencia.

\section{Conclusiones}

La variable alométrica LxA mostró una alta relación con las variables destructivas, por lo cual las regresiones lineales presentaron una alta precisión con las proyecciones del crecimiento en los tallos de pitahaya. Las ecuaciones generadas para cada variable de crecimiento hacen que la herramienta sea más precisa y eficaz para calcular las proyecciones del crecimiento de los tallos. Se propone validar estas herramientas con el objetivo de determinar si pueden sustituir los mecanismos destructivos convencionales del tejido, para cuantificar el crecimiento del cultivo de pitahaya en el trópico seco de Costa Rica.

Se sugiere el uso del LxA para la cuantificación y proyecciones del crecimiento, ya que se pueden utilizar para generar proyecciones de pesos en el tiempo, calcular requerimientos semestrales de fertilización, determinar calidad de semillas de los tallos, calcular áreas fotosintéticamente activas y medir efectos de tratamientos aplicados en el cultivo.

\section{Agradecimiento}

Los autores de este trabajo agradecen el financiamiento a través de los proyectos; VI-520-B7-004 "Análisis de crecimiento de la pitahaya (Hylocereus sp.) en un sistema agroforestal con tutores vivos y el uso de riego por goteo en Santa Cruz, Guanacaste" y VI-520-B6-022 "Curvas de absorción de nutrimentos en pitahaya (Hylocereus sp.) mediante fertilización química y fertilización orgánica en la Finca Experimental Santa Cruz, Guanacaste” y la colaboración del Centro de Investigaciones Agronómicas de la Universidad de Costa Rica.

\section{Literatura citada}

Acosta-Mireles, M., J. Vargas-Hernández, A. Velázquez-Martínez, y J. Etchevers-Barra. 2002. Estimación de la biomasa aérea mediante el uso de relaciones alométricas en seis especies arbóreas en Oaxaca, México. Agrociencia 36:725-736.

Andrade, J., M. Ricalde, L. Simá, C. Cervera, y G. Vargas-Soto. 2006. Microambientes de luz, crecimiento y fotosíntesis de la pitahaya (Hylocereus undatus) en un agrosistema de Yucatán, México. Agrociencia 40:667-697.

Balanguera-López, H., E. Morales, P. Almaza-Merchán, y W. Balaguera. 2010. El tamaño del cladodio y los niveles de auxina influyen en la propagación asexual de pitahaya (Selenicereus megalanthus Haw). Rev. Colom. Cienc. Hort. 4:33-42. doi:10.17584/rcch.2010v4i1.1222

Barthlott, W., K. Burstedde, N. Korotkova, y J. Mutke. 2010. Biodiversidad y distribución de cactáceas. Bol. Soc. Latin. Carib. Cact. Suc. 7(1):1-3.

Bauer, R. 2003. A synopsis of the tribe Hylocereus F. Buxb. Cactaceae Syst. Initiat. 17:3-63. 
Cálix, H., R. Castillo, y H. Caamal. 2014. Caracterización de la producción de pitahaya (Hylocereus spp) en la zona maya de Quintana Roo, México. Agroecología 9:123-132.

Castillo, R., M. Livera, y G. Márquez. 2005. Caracterización morfológica y compatibilidad sexual de cinco genotipos de pitahaya (Hylocereus undatus). Agrociencia 39:183-194.

Cerqueda, H. 2010. Propagación sexual y asexual de la pitahaya (Hylocereus spp). Tesis MSc., Instituto Politécnico Nacional, Ciudad de Mexico, MEX.

Correia, A., R. Roveri, F. Marcelo, D. Mota, and A. Baldo. 2017. Morphological traits as tool to verify genetic variability of interspecific dragon fruit hybrids. Rev. Bras. Frutic. 39(1):e-168. doi:10.1590/0100-29452017168

Esquivel, P., F. Stintzing, and R. Carle. 2007. Comparison of morphological and chemical fruit traits from different pitaya genotypes (Hylocereus sp.) grown in Costa Rica. J. Appl. Bot. Food Qual. 81:7-14.

Esquivel, P., e Y. Araya. 2012. Características del fruto de la pitahaya (Hylocereus spp.) y su potencial de uso en la industria alimentaria. Rev. Venez. Cienc. Tecnol. Alim. 3(1):113-129.

Flores, L. 2011. Indicadores de rentabilidad en la producción de pitahaya (Hylocereus undatus) en San Juan Ixcaquixtla, Puebla. Tesis MSc., Colegio de Postgraduados, Texcoco, Edo. de México, MEX.

Fonseca, W., L. Ruíz, M. Rojas, y F. Alice. 2013. Modelos alométricos para la estimación de biomasa y carbono en Alnus acuminata. Rev. Cienc. Amb. 46(2):37-50. doi:10.15359/rca.46-2.4.

Fumuro, M. 2016. Effects of light intensity, soil acidity, and nitrogen concentration on the vegetative growth of pitaya seedlings $($ C. Acta Hort. 1140:105-112 doi:10.17660/ActaHortic.2016.1140.21.

Galindo, J., y J. Clavijo. 2007. Modelos alométricos para estimar el área de los folios de arvejas (Pisum sativum L.). Rev. Corpoica Cienc. Tecnol. Agropecu. 8(1):37-43. doi:10.21930/rcta.vol8-num1

García, M., y O. Quirós. 2010. Análisis del comportamiento de mercado de la pitahaya (Hylocereus undatus) en Costa Rica. Tecnol. Marcha 23(2):14-24. doi:10.18845/tm.v30i1.3060

Grimaldo-Juárez, O., T. Terrazas, A. García-Velásquez, M. Cruz-Villagas and J.F. Ponce-Medina. 2007. Morphometric analysis of 21 pitahaya (Hylocereus undatus) genotypes. J. PACD 9:99-117.

Gunasena, H., D. Pushpakumara, y M. Kariyawasan. 2007. Dragon fruit Hylocerus undatus Haw Brittin and Rose. In: Pushpakumara et al. editors, Underutilized fruit trees in Sri Lanka. World Agroforestry Centre, New Delhi, IND. p. 110141.

Guzmán, D. 1987. Estudio detallado de suelos y clasificación de uso y aptitud para riego de las tierras de la Finca Experimental Santa Cruz. Tesis Lic., Universidad de Costa Rica, Liberia, CRC.

Huachi, L., E. Yugsi, M. Paredes, D. Coronel, K. Verdugo, y P. Santamaría. 2015. Desarrollo de la pitahaya (Cereus sp) en Ecuador, La Granja. Rev. Cienc. Vida 22(2):50-58. doi:10.17163/lgr.n22.2015.05

Hunt, D., N. Taylor, and G. Charles. 2006. The new cactus lexicon illustrations. 2 ed. dh books, Milborne, GBR.

Juárez, O., A. García, J. Ortiz, y L. Ruiz. 2001 . Características cariotípicas de seis genotipos de pitahaya (Hylocereus spp.) Rev. Chapingo Ser. Hort. 7:177-195. doi:10.5154/r.rchsh.2000.08.056

Juárez, I., F. Ramírez, and T. Cruz. 2009. Caracterización de dos clones de pitahaya roja (Hylocereus purpusii) de Jalisco, México. Rev. Cahpingo Ser. Zonas Áridas 8(1):115-122.

Kishore, K. 2016. Phenological growth stages of dragon fruit (Hylocereus undatus) according to the extended BBCH-scale. Sci. Hortic. 213:294-302. doi:10.1016/j.scienta.2016.10.047 
Le-Bellec, F., and F. Vaillant. 2011. Pitahaya (pitaya) (Hylocereus spp.). In: E. Yahia, editor, Postharvest biology and technology of tropical and subtropical fruits. Vol 4. Mangosteen to white sapote. Woodhead Publishing Limited, Cornwall, GBR. p. 247-273. doi:10.1533/9780857092618.247

López, H., y A. Guido. 2002. Cultivo de la pitahaya. Guía Tecnológica 6. Instituto Nicaragüense de Tecnología Agropecuaria (INTA), NIC.

López, O., y A. Guido. 1998. Evaluación de dosis de nitrógeno y fósforo en el cultivo de la pitahaya (Hylocereus undatus). Agron. Mesoam. 9:66-71. doi:10.15517/am.v9i1.24635

López-Merlín, D., L. Soto-Pinto, G. Jiménez-Ferrer, y S. Hernández-Daumas. 2003. Relaciones alométricas para la predicción de biomasa forrajera y leña de Acacia pennatula y Guazuma ulmifolia en dos comunidades del norte de Chiapas México. Interciencia 28:334-339.

Martín, E., y C. Muñoz. 2017. Métodos para medir la humedad del suelo para la programación del riego ¿Cuándo?: Documento de extensión. University of Arizona, AZ, USA.

Mejía, H., S. Muriel, C. Montoya, and C. Reyes. 2013. In situ morphological characterization of Hylocereus spp. (Fam.: Cactaceae) genotypes from Antioquia and Córdoba (Colombia). Rev. Fac. Nac. Agro. Medellín 66:6845-6854.

Mercado-Silva, E. 2018. Pitaya-Hylocereus undatus (Haw). In: S. Rodrigues et al., editors, Exotic fruits. Academic Press, London, GBR. p. 339-349. doi:10.1016/B978-0-12-803138-4.00045-9

Mizrahi, T., A. Nerd, and P. Nobe. 1997. Cacti as crops. Hort. Rev. 18:291-320.

Mizrahi, Y. 2014. Vine-Cacti pitayas - the new crops of the world. Rev. Bras. Frutic. 36:124-138. doi:10.1590/0100-2945$452 / 13$

Montesinos, J., L. Rodríguez-Larramedi, R. Ortiz-Pérez, M. Fonseca-Flores, G. Ruíz, y F. Guevara-Hernández. 2015. Pitahaya (Hylocereus spp.) un recurso fitogenético con historia y futuro para el trópico seco Mexicano. Cul. Trop. 36(1):67-76.

Morillo, A., Y. Tovar, and Y. Morillo. 2017. Morphological characterization of Selenicereus megalanthus (K. Schum. Ex Vaupel) Moran in the province of Lengupá. Cienc. Desarrollo 7(2):23-33.

Newton, T., J. Abramo, A. Fernández, G. Luis, H. Fogolari, y V. Dos Santos. 2013. Uso do Software ImageJ na estimativa de área foliar para a cultura do feijao. Interciencia 38:843-848.

Nygren, P., S. Rebottaro, and R. Cavarria. 1993. Application of the pipe model theory to non-destructive estimation of leaf biomass and leaf area of pruned. Agrofor. Syst. 23:63-77. doi:10.1007/BF00704851

Ortiz-Hernández; Y., and J. Carrillo-Salazar. 2012. Pitahaya (Hylocereus spp.): a short review. Comun. Sci. 3(4):220-237.

Raveh, E., A. Nerd, and Y. Mizrahi. 1998. Responses of two hemiepiphytic fruit crop cacti to different degrees of shade. Sci. Hort. 73:151-164. doi:10.1016/S0304-4238(97)00134-9

Rincón, N., M.E. Olarte, y J.C. Pérez. 2012. Determinación del área foliar en fotografías tomadas con una cámara web, un teléfono celular o una cámara semiprofesional. Rev. Fac. Nal. Agr. Medellín 65:6399-6402.

Rojas, J., y J. Seminario. 2014. Método alométrico para estimar el área foliar de valeriana (Valeriana pilosa Ruiz y Pav) al estado silvestre. Arnaldoa 21:305-316.

Sabino L. 2010. Relación de las prácticas de manejo con floración de la pitahaya (Hylocereus undatus). Tesis Lic., Instituto Politécnico Nacional, Oaxaca, MEX.

Salazar, N. 1991. Fertilidad de suelos de la Finca Experimental de Santa Cruz (prueba de invernadero) Guanacaste, Costa Rica. Tesis Lic., Universidad de Costa Rica., San José, CRI. 
Sellés, G., R. Ferreyra, G. Contreras, R. Ahumada, J. Valenzuela, y R. Bravo. 2003. Manejo de riego por goteo en Uva de mesa CV. Thompson Seedless cultivada en suelos de textura fina. Agric. Téc. 63:180-192. doi:10.4067/S036528072003000200007

Suárez, R. 2011. Evaluación de métodos de propagación en pitahaya amarilla Selenicereus megalanthus (Haw) Britt and Rose y Pitahaya Roja hylocereus polyrhizus (Haw) britt and Rose. Tesis MSc., Universidad Nacional de Colombia, Bogotá, COL.

Tuan, T. 2012. Vietnam proyecta estabilizar exportación de frutas del Dragón. La voz de Vietnam. VOV MUNDO (VOV 5), VNM. http://vovworld.vn/es-es/Economia/Vietnam-proyecta-estabilizar-exportacion-de-frutas-del-Dragon/98801.vov (consultado 18 mar. 2018).

Vega, E., y R. Salas. 2012. Curvas de absorción de nutrientes bajo dos métodos de fertilización en sandia, en Guanacaste, Costa Rica. InterSedes 13(26):21-42. doi:10.15517/ISUCR.V13I26.2988

Viñas, M., M. Fernánez-Brenes, A. Azofeifa, and V.M. Jiménez. 2012. In vitro propagation of purple pitahaya (Hylocereus costaricensis) [F.A.C. Weber] Britton \& Rose) cv. Cebra. In Vitro Cell. Dev. Biol. Plant 48:469-477. doi:10.1007/s11627012-9439-y

Vaillant, F., A. Perez, I. Davila, M. Dornier, and M. Reynes. 2005. Colorant and antioxidant properties of red-purple pitahaya. Fruits 60:3-12. doi:doi.org/10.1051/fruits:2005007

Yuqing, H., Y. Wiyuan, M. Ling, X. Guangping, Z. Zhongfeng, Z. Danjuan, H. Chengxin, and G. Daxing. 2015. Physiological effect on Hylocereus undulatus and Hylocereus undatus under simulated karst soil water deficiency. J. Resour. Ecol. 6(4):269-275. doi:10.5814/j.issn.1674-764X.2015.04.011 